\title{
The role of long term use of antithyroid drugs in Graves' disease
}

\author{
S A Abhayaratna ${ }^{1}$, N.P. Somasundaram ${ }^{2}$ \\ Sri Lanka Journal of Diabetes, Endocrinology and Metabolism 2013; 3: 41-44
}

\begin{abstract}
Graves' disease is an autoimmune disease characterized by hyperthyroidism due to circulating autoantibodies. Radioactive iodine (RAI) and antithyroid drugs (ATD) are the preferred initial treatment options in Graves' disease in many parts of the world. Long term treatment with methimazole in Grave's disease patients beyond the recommended period is shown to be safe and effective and emerging evidence suggests that continuous methimazole therapy may even be superior to RAl therapy in some aspects. Recent randomized trials comparing long-term methimazole to RAI in patients who relapse, have confirmed these findings.
\end{abstract}

Key words: Graves’ disease, long term antithyroid drugs, radioactive iodine

\section{Graves’ disease}

Graves' disease is an autoimmune disease characterized by hyperthyroidism due to circulating autoantibodies. Thyroid-stimulating immunoglobulins bind and activate thyrotropin receptors, causing the thyroid gland to grow and the thyroid follicles to increase synthesis of thyroid hormone. Untreated Graves' hyperthyroidism may lead to many complications including life threatening thyroid storm.

\section{Treatment options}

$\mathrm{I}^{131}$ therapy (radioactive iodine) and antithyroid drugs are the preferred initial treatment options in Graves' disease in many parts of the world and thyroidectomy is no longer recommended as a first-line therapy. There is a lack of consensus regarding the best treatment for hyperthyroidism. The selection of initial treatment defer from center to center based on availability, cost and patient preference. The current recommendation by The American Thyroid Association (ATA) and American Association of Clinical Endocrinologists (AACE) is, if methimazole is chosen as the primary therapy for Graves' disease, the medication should be continued for approximately 12-18 months and then tapered or discontinued if the TSH is normal at that time (1). The major clinical drawback of treatment with ATD is the high rate of relapse of hyperthyroidism when therapy is discontinued (2). Most of the thyroidologists agree that in patients with recurrent hyperthyroidism, the treatment of choice is radioiodine.

\section{Antithyroid drugs (ATD)}

Graves' hyperthyroidism may be treated with ATD, either alone or in combination with other treatment modalities. The two drugs in common use are methimazole (or its pro-drug carbimazole) and propylthiouracil. They both belong to thionamide group and exert their antithyroid effect by inhibition of thyroid peroxidase catalysed synthesis of thyroid hormones. Thereby thyroid hormone secretion gradually diminishes and the patient becomes euthyroid. An immunosuppressive effect of ATD remains a matter of controversy. The frequency of prolonged remission among patients treated with a thionamides for one to two years varies from 20 to 70 percent with lower rate of remission occurring in children and adolescents(2).

\section{Radioactive iodine (RAI) treatment}

This is the most commonly used treatment modality for Graves’ disease as primary therapy in some countries and as the standard therapy by most when relapse occurs after a course of ATD. RAI is rapidly incorporated into the thyroid cells and its $\beta$-emissions result in extensive local tissue damage. The net effect is ablation of thyroid function over a period of time. ATA and AACE guidelines recommend a dose (typically 10 to $15 \mathrm{mCi}$ ) sufficient to cause hypothyroidism (1). Since there is a probable association between low dose RAI and late occurrence of thyroid cancer in children, relatively high doses of RAI should be administered to minimize residual thyroid tissue (3).

\footnotetext{
${ }^{1}$ Senior Registrar, ${ }^{2}$ Consultant Endocrinologist, National Hospital of Sri Lanka, Colombo.
} 


\section{Limitations of radioactive iodine treatment}

Due to the high failure rate associated with lower doses designed to leave a patient euthyroid, most experts now advocate higher RAI doses which inevitably leads to hypothyroidism, resulting in lifelong surveillance (1). When patients become hypothyroid, they require lifelong replacement with thyroid hormone. Some studies have shown that subclinical hypothyroidism may cause alterations in cognitive function, mood, memory, and general health status which improve with levothyroxine therapy (4). It may also be associated with an increased risk of coronary heart disease (5). However, one study has shown that patients on thyroxine replacement, even with normal TSH levels, demonstrate significant impairment in psychological well-being compared to control individuals (6). If serum TSH concentrations are normal, patients being treated with ATD have fewer complaints than those taking levothyroxine. This might well reflect the ability of the ATD treated patient to continue secreting both T3 and T4. By contrast, serum T3 concentrations in hypothyroid patients depend solely upon the monodeiodination of administered levothyroxine (7). There is increasing evidence that RAI therapy can cause the development or worsening of Graves' ophthalmopathy more often than ATD therapy or surgery and many would avoid it in moderate-to-severe ophthalmopathy. The possibility exists that RAI can precipitate thyroid storm by releasing thyroid hormones, especially in uncontrolled hyperthyroid patients.

Social circumstances in some patients, such as close contact with small children, make them less favourable candidates for RAI. In Sri Lankan setting, RAI is not a readily available option and patients have to be on a long waiting list before receiving treatment free of charge. Due to the cost and scarcity, many patients receive a relatively low dose of RAI (fixed dose of $10 \mathrm{mCi}$ ) which makes them prone to relapse, necessitating a repeat dose. When treated with a lower dose of RAI, many patients have chronic subclinical hyperthyroidism, with associated risks of atrial fibrillation and reduced bone density $(8,9)$.

Although RAI is safe and effective, several large cohort studies have reported increased mortality from vascular causes in radioiodine treated patients $(10,11)$. It has also been proposed that the underlying hyperthyroidism, rather than radioiodine itself, may be responsible for increased vascular mortality in these instances (12).

\section{Surgery}

Surgery is an alternative therapeutic option in patients who are noncompliant or unable to tolerate ATD, have moderate-to-severe ophthalmopathy, large goiters and in patients who refuse or cannot undergo radioiodine therapy. Generally it is reserved for patients with large goiters or goiters with compressive symptoms. It is also indicated in whom the use of ATD and/or RAI does not control hyperthyroidism. Due to cosmetic reasons and perceived risks of surgery and anesthesia, this mode of therapy is not very popular among patients. An experienced surgeon in thyroidectomy is needed to minimize the procedure associated morbidity and mortality. Post operative hypothyroidism will follow total thyroidectomy.

\section{Current evidence for long term use of ATD in patients with Graves' hyperthyroidism}

Search method - To gather evidence for the role of long term ATD in Graves' disease, PUBMED search was done using search terms "Graves disease AND Antithyroid drugs, "Radioiodine OR Long-term methimazole" and "Long-term antithyroid drugs" and filtered by using "Clinical trials", "Humans” and "English”. There were 193, 43 and 46 articles respectively and they were screened for the relevance to the topic. When available, papers that described the results of randomized controlled trials were used and data from case control and cohort studies were included in the absence of randomized trials to supplement information from randomized trials. Eight articles were chosen for the discussion. (3 randomized control trials, 4 cohort studies and 1 case control study).

In adult Graves' disease, the initial duration of ATD was established when it was shown that prolonged initial treatment with ATD was no better than 18 months as far as two year remission rates were concerned (13). It has been the preferred practice to treat with RAI in most patients with Graves' disease (and with surgery where indicated) when they relapse after an initial course of ATD. Some of the limitations with RAI treatment mentioned above have prompted investigators to look into alternative methods in this category of patients. Outcomes and safety of long term use of low dose ATD in patients presenting with a relapse of Grave's disease is of current interest. Some of the studies have particularly looked at this indication of ATD. Due to the associated hepatotoxicity, recent recommendation from ATA and AACE is, propylthiouracil should be administered only in the first trimester of pregnancy and in cases of drug allergy to methimazole (1). Therefore methimazole (or pro-drug carbimazole) is the natural drug of choice in the long term treatment.

Initial retrospective studies of 5-7 years duration of continuous low dose methimazole in Graves' disease made the patients euthyroid, without any major adverse effects. When treated, methimazole prevented relapse mainly in patients above 35 years of age $(14,15)$. 
Two of the recent randomized trials have compared the effectiveness and safety of long term methimazole vs. RAI treatment in relapse of hyperthyroidism after initial course of ATD in adults with diffuse toxic goiter $(16,17)$. In the first study 104 patients over 40 years of age with a diffuse toxic goiter who were initially treated with methimazole and recurred after drug discontinuation, were randomized into 2 groups, continuous methimazole or radioiodine treatment. The results showed that at the end of 10 years, there was no significant difference in duration of symptoms and thyroid function between the two groups. Bone mineral density and echocardiographic measurements were not different between the two groups (16).

The second study which was a more recent and with prolonged follow up, looked into differences in neuropsychological outcome between two modes of treatment in addition to others (17). Out of 239 patients with diffuse toxic goiter who had recurrence of hyperthyroidism after discontinuation of ATD, 104 patients were randomized into two groups, methimazole and treatment with thyroxine following RAI induced hypothyroidism. The remaining 135 patients voluntarily enrolled into either of the two groups. In the RAI group, compared to the methimazoletreated group, during a mean duration of 14 years of follow up, there were more incidences of elevated TSH [> $5 \mathrm{mU} /$ L], increased triglycerides and early diastolic annular velocity, in addition to a decreased early diastolic to annular velocity ratio. The methimazole group scored better in neuropsychology tests that included mood, logical memory, repeated numbers, and intelligence quotient (IQ). BMD was higher in the methimazole-treated group, but the difference did not remain after adjustment for sex. Although prior studies had shown that thyroid stimulating hormone receptor antibody (TRAb) levels are higher following radioiodine, serum concentrations of TRAb antibodies were not significantly different between the groups in this study (17).

Both studies have shown that long term methimazole treatment when compared to RAI in relapse of Graves' disease, is associated with the finding of fewer events of subclinical hypothyroidism, dyslipidemia and lower total cost of treatment. Methimazole treated group had a higher prevalence rate of goiter and presence of thyroid peroxidase (TPO) antibodies in both studies. As in previous retrospective trials, in these randomized studies there were no major side effects associated with long term treatment with methimazole including agranulocytosis or hepatotoxity, reconfirming the safety of the drug in long term use. Some of the findings in the second study (17) with regard to neuropsychological factors, may well be related to intermittent hypothyroidism detected by increases in serum TSH in patients on levothyroxine treatment after radioiodine although a direct role of methimazole on psychological well-being cannot be ruled out.
Optimal treatment of Graves' disease in paediatric patients is still a matter of controversy. Most children are initially treated with ATD. However, it is difficult to achieve long-term compliance and the rate of relapse is high in children. Remission is achieved in less than $30 \%$ of children after a first course of ATD for about 2 years (18). In children, studies have shown that a longer initial duration of euthyroid state with ATD is associated with a lower risk of relapse after a first course of ATD therapy $(18,19)$.

Recently the results of an observational prospective multicenter cohort study with a median follow up of 10 years in 154 newly diagnosed children with Graves'disease, treated with three consecutive courses of carbimazole, each lasting 2 years were published. It suggested that children with Graves' disease who display good compliance with treatment and with no major adverse effects of ATD may be offered up to 8-10 years of medical treatment before definitive treatment is considered (20). As with studies done in adults, long term therapy with carbimazole/ methimazole has been shown to be safe without any major adverse effects $(19,20)$.

\section{Conclusions}

In adult Graves' disease patients with a relapse of hyperthyroidism following an initial course of ATD of optimum duration of 18 months, RAI therapy seems to be the first choice. Though many patients prefer RAI as opposed to long term medications, RAI is not the complete solution to this problem as subclinical or overt hypothyroidism occurs in most RAI treated patients, warranting them to take lifelong thyroxine. Emerging evidence suggests that continuous methimazole therapy may be advantageous over RAI in some aspects and may be considered as an optional method in the long-term treatment of patients with Graves' disease. Those patients who experience recurrence of hyperthyroidism after discontinuation of an effective ATD course, who are willing to take long term treatment and have good compliance are suitable for such an approach. Safety and efficacy of low dose methimazole is clearly shown.

\section{References}

1. Bahn Chair RS, Burch HB, Cooper DS, et al. Hyperthyroidism and other causes of thyrotoxicosis: management guidelines of the American Thyroid Association and American Association of Clinical Endocrinologists. Thyroid 2011; 21: 593.

2. Allannic H, Fauchet R, Orgiazzi J, et al. Antithyroid drugs and Graves' disease: a prospective randomized evaluation of the efficacy of the treatment duration. J Clin Endocrinol Metab 1990; 70: 675-9.

3. Rivkees SA. The management of hyperthyroidism in children with emphasis on the use of radioactive iodine. Pediatr Endocrinol Rev 2003; 2: 212-21. 
4. Samuels MH, Schuff KG, Carlson NE, Carello P, Janowsky JS. Health status, mood, and cognition in experimentally induced subclinical hypothyroidism. J Clin Endocrinol Metab 2007; 92: 2545-51.

5. Rodondi N, den Elzen WP, Bauer DC, et al. Subclinical hypothyroidism and the risk of coronary heart disease and mortality. JAMA 2010; 304: 1365-74.

6. Saravanan P, Chau WF, Roberts N, Vedhara K, Greenwood $\mathrm{R}$, Dayan CM. Psychological well-being in patients on 'adequate' doses of l-thyroxine:results of a large, controlled community-based questionnaire study. Clin Endocrinol (Oxf). 2002; 57: 577-85.

7. Toft AD. Is long-term methimazole therapy as effective as radioiodine for treating hyperthyroidism? Nat Clin Pract Endocrinol Metab 2005; 1(1): 14-5.

8. Sawin CT, Geller A, Wolf PA, Belanger AJ, Baker E, Bacharach P,et al. Low serum thyrotropin concentrations as a risk factor for atrial fibrillation in older patients. $N$ Engl J Med 1994; 331: 1249-52.

9. Faber J, Galloe AM. Changes in bone mass during prolonged subclinical hyperthyroidism due to L-thyroxine treatment: a meta-analysis. Eur J Endocrinol 1994; 130: 350-6.

10. Franklyn JA, Sheppard MC, Maisonneuve P. Thyroid function and mortality in patients treated for hyperthyroidism. JAMA 2005; 294: 71-80.

11. Metso S, Auvinen A, Salmi J, Huhtala H, Jaatinen P. Increased longterm cardiovascular morbidity among patients treated with radioactive iodine for hyperthyroidism. Clin Endocrinol (Oxf). 2008; 68: 450-7.

12. Franklyn JA. Is radioiodine good for hyperthyroidism? Nat Clin Pract Endocrinol Metab 2008; 4: 59.

13. Maugendre D, Gatel A, Campion L et al. Antithyroid drugs and Graves' disease--prospective randomized assessment of long-term treatment. Clin Endocrinol (Oxf). 1999; 50(1): 127-32.

14. Laurberg P, Berman DC, Andersen S, et al. Sustained control of Graves' hyperthyroidism during long-term low-dose antithyroid drug therapy of patients with severe Graves' orbitopathy. Thyroid 2011; 21(9): 951-6.

15. Mazza E, Carlini M, Flecchia D, et al. Long-term followup of patients with hyperthyroidism due to Graves' disease treated with methimazole. Comparison of usual treatment schedule with drug discontinuation vs continuous treatment with low methimazole doses: a retrospective study. $J$ Endocrinol Invest 2008; 31(10): 866-72.

16. Azizi F, Ataie L, Hedayati M, Mehrabi Y, Sheikholeslami F. Effect of long-term continuous methimazole treatment of hyperthyroidism: comparison with radioiodine. Eur $J$ Endocrinol 2005; 152(5): 695-701.

17. Azizi F, Yousefi V, Bahrainian A, Sheikholeslami F, Tohidi M, Mehrabi Y. Long-term Continuous Methimazole or Radioiodine Treatment for Hyperthyroidism. Arch Iran Med 2012; 15(8): 477-84.

18. Kaguelidou F, Alberti C, Castanet M, Guitteny MA, Czernichow P, Le'ger J. Predictors of autoimmune hyperthyroidism relapse in children after discontinuation of antithyroid drug treatment. J Clin Endocrinol Metab 2008; 93: 3817-26.

19. Barrio R, López-Capapé M, Martinez-Badás I, Graves' disease in children and adolescents: response to long-term treatment. Acta Paediatr 2005; 94(11): 1583-9.

20. Leger J, Gelwane G, Kaguelidou F. Positive Impact of Long-Term Antithyroid Drug Treatment on the Outcome of Children with Graves' Disease: National Long-Term Cohort Study. J Clin Endocrinol Metab 2012; 97: 110-19. 